\title{
Carbon Black Nanoparticles Exposure Induces Intestinal Flora Dysbiosis and Consequent Activation of Gut-liver Axis Leading to Liver Lipid Accumulation in Zebrafish
}

\section{Qingqing Liu}

Northeast Agricultural University

\section{Bing Wang}

Northeast Agricultural University

\section{Shengchen Wang}

Northeast Agricultural University

Hongyuan Jing

Northeast Agricultural University

Shiwen Xu ( $\nabla$ shiwenxu@neau.edu.cn)

Northeast Agricultural University

\section{Research}

Keywords: Zebrafish, Carbon black nanoparticles, Intestinal flora, Gut-liver axis, Glycolipid metabolis

Posted Date: August 11th, 2021

DOl: https://doi.org/10.21203/rs.3.rs-792204/v1

License: (1) This work is licensed under a Creative Commons Attribution 4.0 International License. Read Full License 


\section{Abstract}

\section{Background}

Carbon black nanoparticles (CBNPs) are a major carbonaceous nanomaterial, which have been widely left in the environment. The integrity of the gut-liver axis function is critical to the survival of animals. Therefore, we studied the effects of three concentrations of $\operatorname{CBNPs}(50,100,200 \mathrm{mg} / \mathrm{L})$ on zebrafish intestines, liver and intestinal flora.

Results

The results showed that CBNPs exposure could reduce the diversity of intestinal flora, change the structure of core microbial populations, enhance the permeability of the intestinal mucosal barrier, and cause changes in genes related to tight junctions in intestinal tissues. The H\&E staining and Oil red $O$ staining showed that CBNPs exposure would lead to vacuolar degeneration and lipid accumulation in zebrafish liver. Further detection of glycolipid metabolism related genes showed that CBNPs exposure induced the up-regulation of glycolysis related genes PFKFB3, LDHA, and LEPr, reduces the expression of glycogen synthase kinase GSK-3b, and increases lipid transport and production related genes PPAR-a, PPAR-y, LIPC, apoa4, Fabp2 and Fabp11 expression.

\section{Conclusions}

In brief, our data demonstrated that CBNPs exposure induced intestinal microflora disturbance in zebrafish can lead to liver lipid accumulation.

\section{Background}

Carbon black nanoparticles (CBNPs) is a kind of pure black powder separated from the exhaust gas emitted by the combustion of hydrocarbons in the insufficient air(Rodd et al. 2018). Because CBNPs can increase the tensile strength, hardness, tear resistance, abrasion resistance and other properties of rubber products, it is mainly used as rubber reinforcing agent, especially for tires(Mccunney et al. 2012). In addition, the color of carbon black is extremely blackish and the covering power is extremely strong, and it is also an ideal black pigment(Wang et al. 2012). Due to the large-scale production of CBNPs and the expected growth in global demand (for example in the production of tires, rubber, plastics and printing inks), the emissions of CBNPs expand year by year. Studies have shown that CBNPs have obvious toxic effects on animals. For example, after CBNPs invade organisms, they can cause oxidative stress in the organism, affect the structure and function of lysozyme, and also cause lung and systemic inflammation(Ma et al. 2018, Zhang et al. 2019a). At the same time, CBNPs have also been proved to have significant effects on the liver. For example, CBNPs can induce acute inflammation in the liver of mice, damage the liver tissue structure, and cause genotoxicity in the liver(Bourdon et al. 2012, Zhang et al. 2019b).

The gut-liver axis refers to the two-way relationship between the intestine, intestinal microbiota and the liver, which is formed by the integration of signals generated by diet, genetic and environmental factors, and establishes interaction through the portal vein(Albillos et al. 2020, Bortolato et al. 2018). Intestinal mucosal barrier is an important part of the gut-liver axis, it can be used as the site of interaction between the gut and 
liver, which limits the systemic transmission of microorganisms and toxins, and allow nutrients to enter the circulatory system and reach the liver(Camara-Lemarroy et al. 2018). Some environmental crystals and particle irritants have been proved to affect the composition of intestinal microorganisms and cause certain liver damage through the gut-liver axis. For example, oral administration of titanium dioxide nanoparticles can cause mild liver toxicity in rats through the gut-liver axis(Chen et al. 2019). Intravenous injection of biodegradable polylactic acid-glycolic acid nanoparticles can change the intestinal microbiota of obese mouse models and induce reprogramming of the transcriptome in the liver(Chaplin et al. 2020). Polystyrene microplastics exposure can change the intestinal flora function and liver metabolism of marine medaka(Feng et al. 2021). Liver damage caused by the gut-liver axis will impair liver function. And the liver is an important organ of lipid metabolism. As the main regulator of lipid homeostasis, the liver is responsible for coordinating the synthesis, output and subsequent redistribution of fatty acids to other tissues(Nguyen et al. 2008). For example, peroxisome proliferator activated receptor (PPAR) transcription factors can respond to changes in intracellular lipid levels, thereby regulating genes such as Fabp 2 and Fabp 11 that encode fatty acid binding proteins (FABPs) in zebrafish genome, facilitating their combination with saturated, unsaturated and longchain fatty acids, and promoting the uptake and utilization of fatty acids (Esteves et al. 2016, Laprairie et al. 2017, Venkatachalam et al. 2013). Previous studies have shown that intestinal flora disorder caused by harmful substances can lead to liver lipid accumulation. Such as, T.P. Keerthisinghe et al. found that long-term exposure to tetracycline affected the growth of zebrafish, induced intestinal flora disorder and liver lipid accumulation, and significantly up-regulated the expression of apoa4, fabp11 and PPAR(Keerthisinghe et al. 2020). And Zhang et al. found that Microcystin-LR exposure could induce intestinal microbial community transfer in mice, interfere with liver unsaturated fatty acid biosynthesis, and activate the PPAR signaling pathway through overexpression of PPARy, Fabp1 and Fabp2, leading to disorders of liver lipid metabolism(Zhang et al. 2016). Thence, the gut-liver axis has a wide range of significance in the pathogenesis of liver diseases, and has increasingly become the focus of clinical research(Wiest et al. 2018).

In previous studies, laboratories mainly identified CBNPs as air pollutant particles, and explored their toxicological effects on animals through inhalation or intravenous injection. However, due to its extensive production and application, a large amount of industrial wastewater containing CBNPs is continuously discharged into the water environment. Therefore, its toxicological effects on aquatic organisms cannot be ignored. The genes between zebrafish and humans are highly conserved, making them a useful system for studying the basic mechanisms of toxicology (Goessling and Sadler 2015). Therefore, we kept zebrafish in a water environment containing 50,100, and $200 \mathrm{mg} / \mathrm{L}$ CBNPs for 30 days under laboratory conditions and used intestinal, liver and intestinal microbes as the main research objects to evaluate the effect of CBNPs on the growth of zebrafish, the homeostasis of intestinal microflora, the damage of intestinal and liver tissue, as well as related mechanism. These results help to provide useful information on the toxicological effects of CBNPs exposure on zebrafish in aquatic environment, which are essential for formulating effective environmental management and governance policies.

\section{Results}

\subsection{Structural characteristics of CBNPs and the effects of CBNPs exposure on growth status of zebrafish}


The CBNPs (Printex 90) were characterized by TEM and SEM. As shown in Fig. 1A and B, CBNPs appeared in the form of spherical clusters, that was, bubble-like (grape-like) aggregation. The average diameter of the nanospheres in each sample was calculated to be approximately $89 \mathrm{~nm}$.

We continue to monitor the growth and development of juvenile zebrafish after exposure to 50,100 , and 200 $\mathrm{mg} / \mathrm{L}$ CBNPs. Compared with the control group, no obvious deformities were observed in the CBNPs exposure group. Interestingly, the average weight of zebrafish in control group was $0.456 \mathrm{~g}$, CBNPs.L, CBNPs.M and CBNPs.H group were $0.436 \mathrm{~g}, 0.433 \mathrm{~g}, 0.431 \mathrm{~g}$, respectively. Compared with the control group, although the zebrafish body weight of the CBNPs exposure group decreased slightly, it did not show a significant difference, indicating that CBNPs exposure did not affect the zebrafish body weight (Fig. 1C). The body length results showed that after 30 days exposure, the body length of both control group and CBNPs exposure group were about $3.6 \mathrm{~cm}$, which indicated that CBNPs exposure did not affect the zebrafish's body length development (Fig. 1D). Furthermore, according to the formula BMI = weight/height ${ }^{2}$, the zebrafish growth status in each group was quantified. Compared with the control group, the zebrafish body mass index of the CBNPs exposure group did not show a significant difference with the raise of the CBNPs exposure concentration. Token together these results indicate that CBNPs exposure has no effect on the growth and development of zebrafish (Fig. 1E).

\subsection{CBNPs exposure induces zebrafish intestinal tissue damage}

To investigate whether CBNPs exposure can cause damage to zebrafish intestinal tissues, we first performed $\mathrm{H} \& \mathrm{E}$ staining on the zebrafish intestinal tissue. As shown in Fig. 2A, compared with the control group, the intestinal wall of zebrafish intestinal tissue in the CBNPs exposure group became thinner (as indicated by the black arrow), the arrangement of intestinal villi turned to sparse with the raise of CBNPs exposure concentration, the height also changed into shorter, and the lamina propria of intestinal villi increased (as indicated by the red arrow), the number of goblet cells grew in number. Then we observed the microstructure of the intestinal villi by SEM (Fig. 2B). The results showed that compared with the control group, the CBNPs.L, CBNPs.M and CBNPs.H experimental groups showed obvious intestinal injury, such as rough and disordered intestinal surface and disordered intestinal villi arrangement, in which CBNPs.H group could also observe the loss of intestinal villi. We used TEM to further observe the effect of CBNPs exposure on the ultrastructure of zebrafish intestines. As shown in Fig. 2C, compared with the NC group, under the exposure of CBNPs, the zebrafish intestinal tissue had undergone obvious structural changes. It could be observed that the the number of pinocytotic vesicle in intestinal epithelial cells was increased, merged and expanded in all CBNPs groups, and this change was most obvious in the CBNPs.L group. Moreover, as the exposure concentration of CBNPs raised, the tight junction structure was damaged in the CBNPs.M and CBNPs.H groups, and there were gaps between adjacent epithelial cells. These results suggest that CBNPs exposure can cause damage to the intestinal villi, affect the secretory function of the intestinal epithelium and change in intestinal permeability of zebrafish.

\subsection{Effects of CBNPs exposure on the intestinal microflora of zebrafish}




\subsubsection{CBNPs exposure reduces the diversity of zebrafish intestinal flora}

To study the composition of the intestinal flora of each group of zebrafish, OTUs (operational taxonomic units) clustering was performed on the Effective Tags of all samples with $97 \%$ identity. As shown in the Venn diagram based on OTUs in Fig. 3A, the total number of OTUs in all samples was 2236, and there were 276 common OTUs in the four groups. The Venn diagram showed that NC, CBNPs.L, CBNPs.M, and CBNPs.H groups had 1,745, 666, 977, and 548 OTUs, respectively. There were 51, 204 and 107 unique OTUs in CBNPs.L, CBNPs.M, and CBNPs.H groups, respectively, while there were 982 different OTUs in NC group, which was the highest number observed in this study.

After that, we used Alpha Diversity to analyze the microbial community diversity within the sample (Withincommunity). Figure 3B-Figure 3E are respectively the rarefaction curve of the sample, the rank abundance of the sample, the Ace index and PD_whole_tree index box plots. The results showed that compared with the control group, the bacterial diversity of the experimental group decreased. Among them, the CBNPs.L and CBNPs.H groups declined the most obviously $(P<0.05)$. Although the bacterial diversity of CBNPs.M group also reduced, the reduce degree was not significant. Later, we compared and analyzed the composition of microbial communities by Beta diversity, and chose Weighted Unifrac distance and Unweighted Unifrac distance to measure the difference coefficient between the two samples. As shown in Fig. 3F, the results of Beta diversity index thermogram showed that compared with the control group, the difference of species composition in the experimental group increased with the raise of CBNPs exposure concentration. These results indicate that CBNPs at low concentration $(50 \mathrm{mg} / \mathrm{L})$ and high concentration $(200 \mathrm{mg} / \mathrm{L})$ can remarkably affect the microbial diversity in the zebrafish intestine, and the medium concentration of $100 \mathrm{mg} / \mathrm{L}$ CBNPs has little effect on microbial diversity.

\subsubsection{CBNPs exposure changes the species distribution of zebrafish intestinal flora}

After that, we selected the top 10 species with the largest abundance in each group at the phylum level based on the results of species annotations, and generated a columnar cumulative map of species relative abundance. The results were shown in Fig. 4A, the left side was the UPGMA cluster tree structure. From the results, it could be seen that the CBNPs.L and CBNPs.M groups were more similar in microbial composition, followed by the CBNPs.H group, and finally the control group. On the right was the relative abundance distribution of each sample at the phylum level. The results showed that the intestinal flora of the control group was mainly composed of Proteobacteria (45.7\%), Firmicutes (19.9\%), Bacteroidota (17.1\%) and Cyanobacteria (11.4\%). Compared with the control group, the proportion of Proteobacteria in the CBNPs exposure group increased, and the proportions of Proteobacteria in the CBNPs.L group, CBNPs.M group and CBNPs.H group were $68.9 \%, 49.8 \%$ and $91.7 \%$, respectively. And compared with the control group, the proportion of Cyanobacteria was remarkably reduced, and the proportion in the three groups of CBNPs exposure group did not exceed $2 \%$.

The ratio of Firmicutes to Bacteroides (FB) was considered as a biomarker of intestinal diseases. The proportion of Firmicutes in CBNPs.L, CBNPs.M and CBNPs.H groups were $19.3 \%, 26.5 \%$ and 3.9\%, respectively. 
And the proportion of Bacteroidota in the CBNPs.L, CBNPs.M and CBNPs.H groups changed to $2.8 \%, 2.2 \%$ and $0.5 \%$, respectively. According to the ratio of Firmicutes and Bacteroides, the FB ratio was calculated as shown in Fig. 4B. Compared with the control group, the FB ratio of the CBNPs exposure group increased remarkably ( $P$ $<0.05)$, and the CBNPs.M group increased the most remarkably $(P<0.01)$.

In addition, in order to understand the characteristics of different abundances and related categories between the groups, the results of LEfSe (LDA Effect Size) analysis $(P<0.05$, LDA $>4)$ were shown in Fig. 4C, which were respectively histograms of LDA value distribution and the evolutionary branch diagram. The histogram of LDA value distribution on the left showed that 37 species had significant differences between the groups from the phylum to the genus level. Compared with the group, CBNPs.M group with medium concentration could cause the largest number of obvious changes in the group, compared with the group, CBNPs.M group with medium concentration could cause the largest number of obvious changes in the group, including C_Alphaproteobacteria, o_Rhizobiales, f_Xanthobacteraceae, g_Afipia and nine other flora. CBNPs exposure at low concentrations caused a total of six groups to change, including g_Enterobacter, f_Enterobacteriaceae, o_Enterobacterales, and three other flora. Exposure to high concentrations of CBNPs resulted in changes in six taxa, including f_Aeromonadaceae, c_Gammaproteobacteria and their subtypes. The evolutionary branch diagram on the right showed that Bacteroidota and Limnochordia played important roles in the control group, the CBNPs.L group was the conditional pathogen of Enterobacteraies, and the CBNPs.M group was mainly the pathogenic bacteria of Actinobacteria and Alphaproteobacteria, CBNPs.H group was the pathogenic bacteria genus of Gammaproteobacteria. The above results all indicate that CBNPs exposure can affect the composition of intestinal microorganisms in zebrafish, and the composition of intestinal microorganisms varies according to different exposure concentrations.

\subsection{CBNPs exposure induces liver damage and lipid accumulation in zebrafish}

Next, we performed histopathological analysis of zebrafish liver tissue. The representative microscopic images of H\&E staining liver tissue sections showed that compared with the control group, the cells in the CBNPsexposed group had obvious vacuolar degeneration (Fig. 5A). As indicated by the arrow, the cell body was evidently enlarged, the cytoplasm was blank, and the nucleus was squeezed to one side. However, no significant differences were seen between the exposure groups with different CBNPs concentrations.

Subsequently, TEM was used to observe the changes in the microstructure of liver tissue. As shown in Fig. 5B, it could be observed that compared with the control group, the volume of hepatocytes in the CBNPs exposed group enlarged, the cytoplasm was empty and transparent, the intracellular organelles decreased obviously and disappeared, only a small amount of mitochondria and other organelles remained near the cell membrane and nuclear membrane, which was consistent with the results of $\mathrm{H}$ \& $\mathrm{E}$ staining. It could be identified that with the raise of CBNPs exposure concentration, the organelle loss of hepatocytes was more serious, indicating that the liver injury was aggravated.

Later, we examined the changes of lipid accumulation in the liver by Oil Red $\mathrm{O}$ staining in order to investigate whether the liver injury caused by CBNPs exposure would affect the liver function. As shown in Fig. 5C, a small amount of lipid droplets were observed in the control group. Compared with the control group, the content of lipid droplets in the liver increased with the raise of CBNPs concentration in the CBNPs exposed group. This 
result shows that CBNPs exposure can affect the liver lipid metabolism of zebrafish and cause lipid accumulation.

\subsection{CBNPs exposure leads to increased permeability of zebrafish intestinal tissues}

RT-PCR technology was used to detect the mRNA expression levels of tight junction-related genes (Occludin, ZO-1, Rap2, RhoA, ROCK, and MLC2) in zebrafish intestinal tissues, and the results were showed in Fig. 6A. Compared with the control group, the expressions of tight junction related genes in the low concentration CBNPs.L group were basically unchanged $(P>0.05)$. The mRNA expressions of Occludin, ZO- 1 and Rap2 in the medium and high concentration CBNPs.M and CBNPs.H groups were down-regulated, while the expressions of RhoA, ROCK and MLC2 were up-regulated $(P<0.05)$, which was consistent with the results of transmission electron microscopy The above results indicate that CBNPs can change the permeability of the zebrafish intestinal mucosal barrier under exposure to environmental concentrations of $100 \mathrm{mg} / \mathrm{L}$ and $200 \mathrm{mg} / \mathrm{L}$.

\subsection{CBNPs exposure causes disorder of glycolipid metabolism in zebrafish liver}

To investigate the effect of CBNPs on the liver function of zebrafish, we detected the expression changes of glycolysis and lipid metabolism related genes in zebrafish liver tissue. As shown in Figure. 6B, compared with the control group, the mRNA expressions of glycolysis-related genes PFKFB3, LDHA, and LEPr upregulated in the CBNPs exposure group $(P<0.05)$, while the mRNA expression of GSK-3b decreased remarkably only in CBNPs.H group $(P<0.05)$. In addition, the mRNA expressions of lipid metabolism-related genes PPAR-a, PPARY, LIPC, apoa4, Fabp2, and Fabp11 promoted in the CBNPs exposure group $(P<0.05)$. Figure $6 \mathrm{C}$ showed the results of protein expression levels of glycolysis and lipid metabolism-related genes in zebrafish liver tissue, and its trend was basically consistent with the changing trend of mRNA expression. The above results are consistent with the results of oil red $\mathrm{O}$ staining, indicating that CBNPs exposure can cause disorders of glycolipid metabolism in zebrafish liver tissues and cause lipid accumulation.

\section{Discussion}

Carbon black nanoparticles (CBNPs) are carbon-containing nanomaterials mainly used as industrial raw materials(Vance et al. 2015). Due to their reinforcing properties to rubber, they are widely used in rubber and tire manufacturing, and with the continuous grow in global production. Abandoned CBNPs have been widely distributed in the environment and pose potentially toxic effects on a variety of organisms(Surya et al. 2015). Here, in order to explore the possible toxic effects of CBNPs exposure on the gut-liver axis of aquatic organisms, we used the water quality test model animal zebrafish as the test sample to examine the changes of gut, liver and intestinal flora after exposure to CBNPs. The results showed that CBNPs exposure did not affect the growth and development of zebrafish, but could reduce the diversity of intestinal flora, change the core microflora, damage the intestinal villi, increases the permeability of the intestinal mucosal barrier, cause vacuolar degeneration of liver, and finally lead to liver glycolipid metabolism disorder and lipid accumulation. 
A healthy gut microbial ecosystem is a necessary condition for maintaining the orderly functions of the organism. In recent years, the researches on most inorganic and metal nanoparticles have shown that the biocidal activity of nanoparticles may promote the chronic changes of the composition and/or metabolic activities of symbiotic bacteria (i.e. intestinal flora imbalance)(Lamas et al. 2020). Oral administration of silver nanoparticles at relevant doses in humans could cause changes in mouse gut microbes and interfere with the uniformity (a diversity) and population ( $\beta$ diversity) of bacteria in a dose-dependent manner(van den Brule et al. 2016). Copper oxide nanoparticles interfered with the intestinal microbial community structure of soil collembolas and reduced the diversity of intestinal microbes(Ding et al. 2020). After 7 days and 14 days of oral administration of TiO nanoparticles in weaned rats, inflammation and infiltration damage of colonic epithelial tissue was observed, and the intestinal microbiota fluctuated with the decrease in the abundance of Lactobacillus in feces(Zhao et al. 2020). In this experiment, we found that CBNPs at $50 \mathrm{mg} / \mathrm{L}$ and $200 \mathrm{mg} / \mathrm{L}$ can obviously affect the microbial diversity in zebrafish intestine, and $100 \mathrm{mg} / \mathrm{L}$ CBNPs exposure had little effect on microbial diversity. At the same time, CBNPs exposure changed the structure of the core microbial population in each group, evidently reduced the proportion of beneficial bacteria Bacteroidota in the experimental group, and increased the abundance of Proteobacteria as a marker of bacterial imbalance. The above results indicate that CBNPs exposure can reduce the diversity of zebrafish intestinal flora, change the composition of the flora, thus resulting in intestinal flora imbalance.

The intestinal mucosal barrier is an important part of the gut-liver axis, including the mucous layer containing symbiotic microorganisms, the epithelial intestinal layer tightly connected between adjacent cells, and the lamina propria containing innate tissues and adaptive immune cells(Bajaj 2019). The control of the microbial community is essential for maintaining the homeostasis of the gut-liver axis. The damage of intestinal mucosal barrier function caused by the abnormal or imbalance of the intestinal microbial composition can increase intestinal permeability(Tripathi et al. 2018). It has been found that long-term high-protein diet feeding could change the diversity of the intestinal flora of mice, increase intestinal permeability, and cause systemic inflammation and kidney damage(Snelson et al. 2021). Exposure to 1-octyl-3-methylimidazole hexafluorophosphate could obviously reduce the intestinal microbial diversity of carp, and damage the intestinal physical barrier and immune barrier by reducing the carp intestinal villi height and the mRNA expression of tight junction genes(Chang et al. 2020). And the intestinal pathogenic bacteria PSaV could induce reduction of transepithelial resistance, increase of paracellular flow, intracellular translocation of occlusion and lateral membrane lipid diffusion by activating the RhoA/ROCK/MLC pathway, thereby destroying tight junctions of epithelial cells(Sharif et al. 2021). In our study, CBNPs exposure caused zebrafish intestinal villi damage and reduced the height of intestinal villi. As the exposure concentration of CBNPs increased, tight junction structure damage was observed in the CBNPs.M and CBNPs.H groups and the expression of genes related to tight connection was changed. The results show that medium concentration ( $100 \mathrm{mg} / \mathrm{L})$ and high concentration $(200 \mathrm{mg} / \mathrm{L})$ CBNPs can destroy the tight junctions between the intestinal epithelium and increase the permeability of the intestinal mucosal barrier.

Intestinal flora disorder-induced the increase of intestinal permeability can cause liver glycolipid metabolism disorder via liver-gut axis. It was reported that Bisphenol A exposure caused intestinal flora imbalance, enhanced intestinal permeability and endotoxin level, and then activated gut-liver axis, leading to liver steatosis in CD-1 mice(Feng et al. 2020). And chlorothalonil and procymidone and their combined administration can change the structure of the intestinal flora of mice, destroy the integrity of the intestinal barrier function, and 
affect liver glycolipid metabolism(Wang et al. 2021). In this study, we found that CBNPs exposure increased the ratio (F/B) of Firmicutes and Bacteroides, which is considered to be related to body obesity, and cause liver cell damage and lipid accumulation in liver. The liver plays an important role in coordinating the homeostasis of systemic metabolism and the supply and lack of nutrients. Liver damage can cause changes in its functions. For instance, thiafluromide exposure could cause liver damage in adult zebrafish and affect the expression of glucose metabolism related genes(Yang et al. 2017). Environmentally relevant concentrations of microcystins could cause liver damage and affect lipid metabolism in zebrafish(Chen et al. 2017). This study further detected lipid and glycolysis-related genes and found that CBNPs exposure upregulated the expression of glycolysis related genes, downregulate the expression of glycogen synthase kinase, and increased the expression of lipid transport and production related genes, confirming that CBNPs exposure cause disorders of glycolipid metabolism in zebrafish liver. This result is also consistent with the finding of lipid accumulation in liver, indicating that CBNPs exposure can cause liver lipid accumulation by affecting glycolipid metabolism.

\section{Conclusions}

In summary, CBNPs exposure can reduce the diversity of zebrafish intestinal flora, change the composition of flora, change the expression of tight junction-related genes, increase the permeability of the intestinal mucosal barrier, and cause liver damage through the gut-liver axis, resulting in disorder of glycolipid metabolism and lipid accumulation. This study shows that exposure to CBNPs has a certain potential risk to zebrafish. The research results are helpful to study the toxic effects of CBNPs on aquatic ecosystems, and have certain reference significance for the health risk assessment of related environmental particle exposure.

\section{Materials And Methods}

\subsection{CBNPs physiochemical characterization}

Commercial CBNPs (Printex-90, Jinan Kasong Chemical Co., Ltd, China) were dispersed in anhydrous ethanol, and the concentration was adjusted to $1 \mathrm{mg} / \mathrm{ml}$. Then ultrasonic treatment was carried out for 1 hour with $100 \mathrm{~Hz}$ and $200 \mathrm{~W}$ ultrasonic cleaning machine (Kunshan ultrasonic cleaner in Jiangsu Province, China), and water was changed every 15 minutes. After the CBNPs solution was fully dispersed, the structure of CBNPs was observed by transmission electron microscope (TEM, Hitachi, HT7800, Japan) and scanning electron microscope (SEM, Hitachi, SU8010, Japan).

\subsection{Treatment of experimental animals}

360 3-month-old zebrafish were randomly divided into four groups, the CBNPs concentration 50mg/L group (CBNPs.L), 100mg/L group (CBNPs.M), 200mg/L group (CBNPs.H) and the control group (NC), (90 per group). Before experiment, all zebrafish were fed with juvenile shrimp or brine shrimp every day in a controlled laboratory environment $\left(28 \pm 1^{\circ} \mathrm{C}, 14 \mathrm{~h}\right.$ light/10 h dark rearing cycle, humidity $\left.80 \%\right)$. According to the allowable CBNPs emission requirements in the "Integrated Wastewater Discharge Standard" (GB 8978 - 1996), the minimum exposure concentration of CBNPs in the experimental group was $50 \mathrm{mg} / \mathrm{L}$, and the other two groups were set at $100 \mathrm{mg} / \mathrm{L}$ and $200 \mathrm{mg} / \mathrm{L}$ according to the concentration gradient. In the experimental group, we used circulating water to control the final concentration of CBNPs in the water environment of each group, and updated the environmental water every 5 days. After 30 days of CBNPs exposure, four groups of zebrafish liver 
and intestine were collected, rinsed with ice PBS and fixed in $4 \%$ paraformaldehyde (PFA) or stored at $-80{ }^{\circ} \mathrm{C}$ for further analysis. The intestinal contents were collected in a sterile environment, and the samples were quickly placed in liquid nitrogen, and then immediately stored at $-80^{\circ} \mathrm{C}$. All procedures were approved by the Northeast Agricultural University Animal Management and Use Committee (SRM-11).

\subsection{Observation of the microstructure of liver and intestinal tissues}

For hematoxylin-eosin (H\&E) staining, part of liver and intestinal tissues were taken out from 4\% PFA solution, rehydrated and dehydrated in ethanol, permeabilized in xylene, embedded in paraffin, and finally made into paraffin blocks. After sectioning, the sections were stained according to the H\&E staining method(Zhao et al. 2021). The images were obtained by microscope (Olympus, Japan). The remaining liver tissue was taken out from 4\% PFA solution, frozen section was made after rapid freezing, and then stained according to oil red 0 staining method(Shengchen et al. 2021). The images were obtained by microscope.

For ultramicroscopic observation of tissues, the liver and intestinal tissues were taken out of the $2.5 \%$ glutaraldehyde solution, and part of them were prepared by standard methods for SEM observation(Chi et al. 2021b). Before observation, the samples were sputtered with gold powder (E-1010, HITACHI), and observed with S-3400N (HITACHI) scanning electron microscope. The other part was prepared by standard method and observed by TEM(Hu et al. 2019).

\subsection{S bioinformatics analysis of intestinal flora}

The zebrafish intestinal contents were analyzed for intestinal flora. This sequencing experiment was completed by Beijing Nuohe Zhiyuan Technology Co., Ltd. DNA extraction and detection were performed on the collected samples, and then PCR amplification of the V3 + V4 region was performed according to the primer sequence in Table 1. After purification, library preparation and library examination were carried out, and finally the sequencing was carried out.

After sequencing, perform information analysis. Firstly, the original data was spliced and filtered to obtain effective data (Clean Data), and then based on the effective data, OTUs (Operational Taxonomic Units) clustering and species classification analysis were performed, and the representative sequence of each OTU was made species Annotate, analyze the abundance, Alpha diversity calculation, Venn map and petal map of OTUs, perform the difference significance test, and finally perform the function prediction analysis.

\subsection{RNA extraction and quantitative real-time PCR (qRT-PCR)}

Total RNA was extracted from zebrafish liver and intestinal tissues by Trizol method(Chi et al. 2021a). We used NanoDrop ND-2000 (Thermo Fisher Scientific) to detect the extracted RNA, when A260/A280 was between 1.9 and 2.1, the extracted RNA can be used for subsequent first strand cDNA synthesis. Perform reverse transcription according to the manufacturer's instructions (BIOER, Hangzhou, China). QRT-PCR was used to detect the expression level of related genes, and the LineGene9600Plus detection system (BIOER, Hangzhou, China) was used for qRT-PCR detection. The primers of the tested genes were shown in Table 2. According to the $2^{-\Delta \Delta C t}$ method, compared with the expression of the housekeeping gene $\beta$-actin to calculate the relative expression of the target gene. 
Table 1

\begin{tabular}{|lll|}
\hline Region & Primers & Primer sequence $\left(\mathbf{5}^{\prime} \rightarrow \mathbf{3}^{\prime}\right)$ \\
\hline V3 + V4 & $341 \mathrm{~F}$ & CCTAYGGGRBGCASCAG \\
\cline { 2 - 3 } & $806 \mathrm{R}$ & GGACTACNNGGGTATCTAAT \\
\hline Gene-special primers used in the real-time quantitative reverse transcription PCR. \\
\hline
\end{tabular}

Table 2

\begin{tabular}{|c|c|c|c|}
\hline Gene & Forward primer $\left(5^{\prime} \rightarrow 3^{\prime}\right)$ & Reverse primer $\left(5^{\prime} \rightarrow 3^{\prime}\right)$ & Serial number \\
\hline LDHA & TCTGCTCGTTTCCGCTACTTGATG & CCAGCCACATTCACACCACTCC & NM_131246.1 \\
\hline LIPC & GGATTCGAGCAGACGGTCAAGTG & TTCTTTCGGCAGTCCAGACAGTTG & NM_201022.1 \\
\hline GSK3b & GTGCTGGCTGAACTGCTGCTC & TGCGGGAATTTGAACTCGGTGTAG & XM_009304494.3 \\
\hline PFKFB3 & TGATTGATGTCGGTCGCAGATTCC & CCTGGAGGTTGTGCTGGCTTTC & NM_213397.1 \\
\hline LEPr & САССАТССТССТСТCCGACCAG & TCCGAGTTGTTGGCAGAGAAGTTG & NM_001309403.1 \\
\hline Occludin & TGTGTGCTGTCCATCATCTTCTGC & CGCTGCCATAGGAACCACCATAG & NM_212832.2 \\
\hline ZO-1 & ACGGAGTCTGTGGAGAAGGTGAG & GGTGAGTTTGGGCAGGGATGTG & XM_021470723.1 \\
\hline Rap2c & CATCAGACCAATGCGGGACCAG & CGACCATCAGAACCAGCAACCTC & NM_001007055.1 \\
\hline RhoA & CAGGGCAGGAGGACTACGACAG & TGACCTCAGGCGTCCACTTCTC' & NM_212749.2 \\
\hline ROCK & GGACCAGAAAGCCCAGAAGAAACC & TTCTCACCCTCGCCCGCATAG & NM_174863.2 \\
\hline MLC2 & CGTCATCGTGTCTGCCTTCAAGG & TCTCCTCTGCGGTGAACCTGTC & NM_131188.2 \\
\hline Fabp2 & АCTTTACTCTGGGCGTCACCTTTG & TCCGTTGTCCTTGCGTGTGAAAG & NM_131431.1 \\
\hline Fabp11 & GACGGCAAGGAGTCCACAATAGAG & GACAGCCACCACATCACCCATC & NM_001004682.1 \\
\hline apoa4 & CGCCGTCACCCTCAAGAAACAG & TCCCTCAGCACTTCAGTCTCCTTG & NM_001079861.1 \\
\hline PPAR-a & TCGGATCGGGCATCAGGATACC & ACAGCGGCGTTCACACTTATCG & NM_001102567.1 \\
\hline PPAR-Y & GACACGCACTCGCTGGACATC & GGGTTGGGTCATTCTGTGTTGGG & NM_131467.1 \\
\hline$\beta$-actin & GGCTCTCTTCCAGCCTTCCT & AGCACGGTGTTGGCATACAG & NM_131031.2 \\
\hline
\end{tabular}

\subsection{Protein extraction and western blot detection}

The Western blot procedures were performed as described before(Zhang et al. 2020). In brief, Western and IP cell lysate (Beyotime, Shanghai, China) and Phenylmethylsulfonyl fluoride (PMSF, Beyotime, Shanghai, China) were used to extract the total protein of each group of tissue cells, and the concentration and purity were determined. After that, $1 / 4$ amount of SDS was added, boiled for 10 minutes, and stored at $-20^{\circ} \mathrm{C}$. The total protein was loaded on SDS polyacrylamide gel electrophoresis, separated by electrophoresis, and then transferred to nitrocellulose membrane. The membrane was sealed with $5 \%$ skim milk at $37^{\circ} \mathrm{C}$ for $2 \mathrm{~h}$ and 
incubated overnight with the corresponding dilution of primary antibody at $4{ }^{\circ} \mathrm{C}$. The dilution factor of the primary antibody was shown in Table 2. Then wash three times in PBST, incubate with secondary antibody, and wash three times in TBST. The protein bands were detected by Ultra-sensitive ECL chemiluminescence solution (Lanjieco Technology Co., Ltd.) and chemiluminescence imaging system (Azure Biosystems, USA). The relative abundance of proteins was standardized as $\beta$-actin. The band intensity was quantified by the mean pixel intensity using Image $\mathrm{J}$ software.

\subsection{Data Analysis}

Statistical analysis of all data was carried out with one-way analysis of variance (ANOVA) using GraphPad Prism Version 8.0 software. All the experiments were repeated at least three times and the values were expressed as the "mean \pm SD". There were significant differences between two or more groups with different letters, $P<0.05$; the same letter indicated that there was no significant difference between different groups, $P>$ 0.05 .

\section{Abbreviations}

\begin{tabular}{|ll|}
\hline apoa4 & Recombinant Apolipoprotein A4 \\
\hline BMI & Body mass index \\
\hline CBNPs & Carbon black nanoparticles \\
\hline Fabp11 & Fatty acid-binding protein 11 \\
\hline Fabp2 & Fatty acid-binding protein 2 \\
\hline FABPs & fatty acid binding proteins \\
\hline H\&E & hematoxylin-eosin \\
\hline LIPC & Hepatic lipase (gene) \\
\hline MLC2 & Myosin light-chain 2 \\
\hline PBST & Phosphate buffered solution \\
\hline PFKFB3 & 6-phosphofructokinase-2/fructose-2,6-bisphosphatase 3 \\
\hline PMSF & Phenylmethylsulfonyl fluoride \\
\hline PPAR & peroxisome proliferator activated receptor \\
\hline RhoA & Ras homolog family member A \\
\hline ROCK & Rho-associated kinase \\
\hline SDS & Sodium dodecyl sulfate \\
\hline SEM & Scanning electron microscope \\
\hline TCA & Tricarboxylic acid cycle \\
\hline UPGMA & Unweighted pair-group method with arithmetic means \\
\hline ZO-1 & Zonula occludens-1 \\
\hline
\end{tabular}

Page $12 / 22$ 


\section{Declarations}

\section{Ethics approval and consent to participate}

All procedures were approved by the Northeast Agricultural University Animal Management and Use Committee (SRM-11).

\section{Consent for publication}

Not applicable.

\section{Availability of data and materials}

The data used to support the findings of this study are available from the corresponding author upon request.

\section{Competing interests}

The authors declare that they have no known competing financial interests or personal relationships that could have appeared to influence the work reported in this paper.

\section{Funding}

This research did not receive any specific grant from funding agencies in the public, commercial, or not-forprofit sectors.

\section{Authors' contributions}

Shiwen Xu provided ideas for the experiment and experimental supplies. Qingqing Liu completed the experiment and wrote the manuscript. Bing Wang and Hongyuan Jing participated in some experiments, and Shengchen Wang revised and polished the manuscript.

\section{Acknowledgements}

Not applicable.

\section{References}

Albillos, A., de Gottardi, A. and Rescigno, M. (2020) The gut-liver axis in liver disease: Pathophysiological basis for therapy. Journal of Hepatology 72(3), 558-577.

Bortolato, M., Floris, G. and Shih, J.C. (2018) From aggression to autism: new perspectives on the behavioral sequelae of monoamine oxidase deficiency. Journal of Neural Transmission 125(11), 1589-1599.

Bourdon, J.A., Saber, A.T., Jacobsen, N.R., Jensen, K.A., Madsen, A.M., Lamson, J.S., Wallin, H., Moller, P., Loft, S., Yauk, C.L. and Vogel, U.B. (2012) Carbon black nanoparticle instillation induces sustained inflammation and genotoxicity in mouse lung and liver. Particle and fibre toxicology 9. 
Camara-Lemarroy, C.R., Metz, L., Meddings, J.B., Sharkey, K.A. and Yong, V.W. (2018) The intestinal barrier in multiple sclerosis: implications for pathophysiology and therapeutics. Brain 141, 1900-1916.

Chaplin, A., Gao, H., Asase, C., Rengasamy, P., Park, B., Skander, D., Bebek, G., Rajagopalan, S. and Maiseyeu, A. (2020) Systemically-delivered biodegradable PLGA alters gut microbiota and induces transcriptomic reprogramming in the liver in an obesity mouse model. Scientific Reports 10(1).

Chen, Z., Zhou, D., Han, S., Zhou, S. and Jia, G. (2019) Hepatotoxicity and the role of the gut-liver axis in rats after oral administration of titanium dioxide nanoparticles. Particle and fibre toxicology 16(1).

Chi, Q., Hu, X., Zhao, B., Zhang, Q., Zhang, K. and Li, S. (2021a) Regulation of H2S-induced necroptosis and inflammation in broiler bursa of Fabricius by the miR-15b-5p/TGFBR3 axis and the involvement of oxidative stress in this process. Journal of Hazardous Materials 406.

Chi, Q., Zhang, Q., Lu, Y., Zhang, Y., Xu, S. and Li, S. (2021b) Roles of selenoprotein S in reactive oxygen speciesdependent neutrophil extracellular trap formation induced by selenium-deficient arteritis. Redox biology 44, 102003-102003.

Esteves, A., Knoll-Gellida, A., Canclini, L., Silvarrey, M.C., Andre, M. and Babin, P.J. (2016) Fatty acid binding proteins have the potential to channel dietary fatty acids into enterocyte nuclei. Journal of Lipid Research $57(2), 219-232$.

Feng, S., Zeng, Y., Cai, Z., Wu, J., Chan, L.L., Zhu, J. and Zhou, J. (2021) Polystyrenemicroplastics alter the intestinalmicrobiota function and the hepatic metabolism status in marine medaka (Oryzias melastigma). Science of the Total Environment 759.

Goessling, W. and Sadler, K.C. (2015) Zebrafish: An Important Tool for Liver Disease Research. Gastroenterology 149(6), 1361-1377.

Hu, X., Chi, Q., Liu, Q., Wang, D., Zhang, Y. and Li, S. (2019) Atmospheric H2S triggers immune damage by activating the TLR-7/MyD88/NF-kappa B pathway and NLRP3 inflammasome in broiler thymus. Chemosphere 237.

Keerthisinghe, T.P., Wang, F., Wang, M., Yang, Q., Li, J., Yang, J., Xi, L., Dong, W. and Fang, M. (2020) Long-term exposure to TET increases body weight of juvenile zebrafish as indicated in host metabolism and gut microbiome. Environment International 139.

Laprairie, R.B., Denovan-Wright, E.M. and Wright, J.M. (2017) Differential regulation of the duplicated fabp7, fabp10 and fabp11 genes of zebrafish by peroxisome proliferator activated receptors. Comparative Biochemistry and Physiology B-Biochemistry \& Molecular Biology 213, 81-90.

Ma, J., Guo, A., Wang, S., Man, S., Zhang, Y., Liu, S. and Liu, Y. (2018) From the lung to the knee joint: Toxicity evaluation of carbon black nanoparticles on macrophages and chondrocytes. Journal of Hazardous Materials 353, 329-339. 
Mccunney, R.J., Muranko, H.J., Long, C.M., Hamade, A.K. and Morfeld, P. (2012) Carbon Black, Patty's Toxicology.

Nguyen, P., Leray, V., Diez, M., Serisier, S. and Dumon, H. (2008) Liver lipid metabolism. Journal of Animal Physiology \& Animal Nutrition 92(3), 272-283.

Rodd, A.L., Castilho, C.J., Chaparro, C.E.F., Rene Rangel-Mendez, J., Hurt, R.H. and Kane, A.B. (2018) Impact of emerging, high-production-volume graphene-based materials on the bioavailability of benzo(a)pyrene to brine shrimp and fish liver cells. Environmental Science-Nano 5(9), 2144-2161.

Shengchen, W., Jing, L., Yujie, Y., Yue, W. and Shiwen, X. (2021) Polystyrene microplastics-induced ROS overproduction disrupts the skeletal muscle regeneration by converting myoblasts into adipocytes. Journal of Hazardous Materials 417, 125962-125962.

Venkatachalam, A.B., Sawler, D.L. and Wright, J.M. (2013) Tissue-specific transcriptional modulation of fatty acid-binding protein genes, fabp2, fabp3 and fabp6, by fatty acids and the peroxisome proliferator, clofibrate, in zebrafish (Danio rerio). Gene 520(1), 14-21.

Wang, C., Zhang, X., Lv, F. and Peng, L. (2012) Using carbon black nanoparticles to dye the cationic-modified cotton fabrics. Journal of Applied Polymer Science 124(6), 5194-5199.

Wiest, R., Albillos, A., Trauner, M., Bajaj, J.S. and Jalan, R. (2018) Targeting the gut-liver axis in liver disease (vol 67, pg 1084, 2017). Journal of Hepatology 68(6), 1336-1336.

Zhang, R., Jia, C., Zhao, L., Pan, J., Niu, Q. and Liu, R. (2019a) Characterization of the interaction between carbon black and three important antioxidant proteins using multi spectroscopy and modeling simulations. Chemosphere 222, 823-830.

Zhang, R., Zhang, X., Gao, S. and Liu, R. (2019b) Assessing the in vitro and in vivo toxicity of ultrafine carbon black to mouse liver. Science of the Total Environment 655, 1334-1341.

Zhang, Z., Liu, Q., Yang, J., Yao, H., Fan, R., Cao, C., Liu, C., Zhang, S., Lei, X. and Xu, S. (2020) The proteomic profiling of multiple tissue damage in chickens for a selenium deficiency biomarker discovery. Food \& Function $11(2), 1312-1321$.

Zhang, Z., Zhang, X.-X., Wu, B., Yin, J., Yu, Y. and Yang, L. (2016) Comprehensive insights into microcystin-LR effects on hepatic lipid metabolism using cross-omics technologies. Journal of Hazardous Materials 315, 126134.

Zhao, H., Wang, Y., Guo, M., Liu, Y., Yu, H. and Xing, M. (2021) Environmentally relevant concentration of cypermethrin or/and sulfamethoxazole induce neurotoxicity of grass carp: Involvement of blood-brain barrier, oxidative stress and apoptosis. Science of the Total Environment 762.

\section{Figures}


A

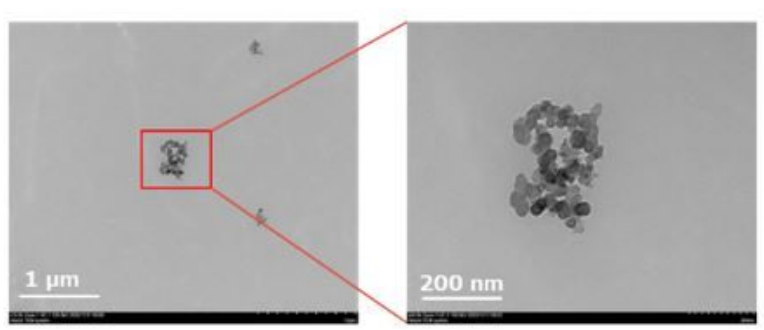

B
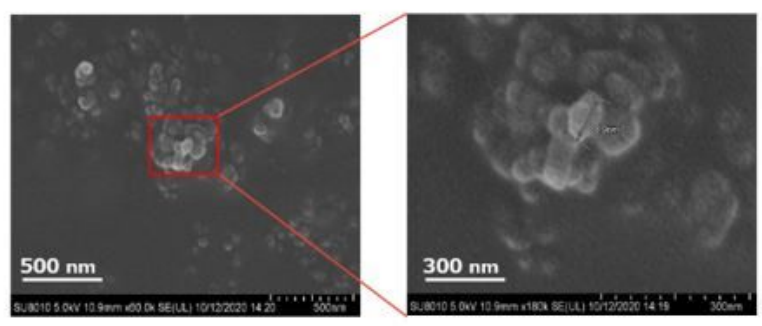

C

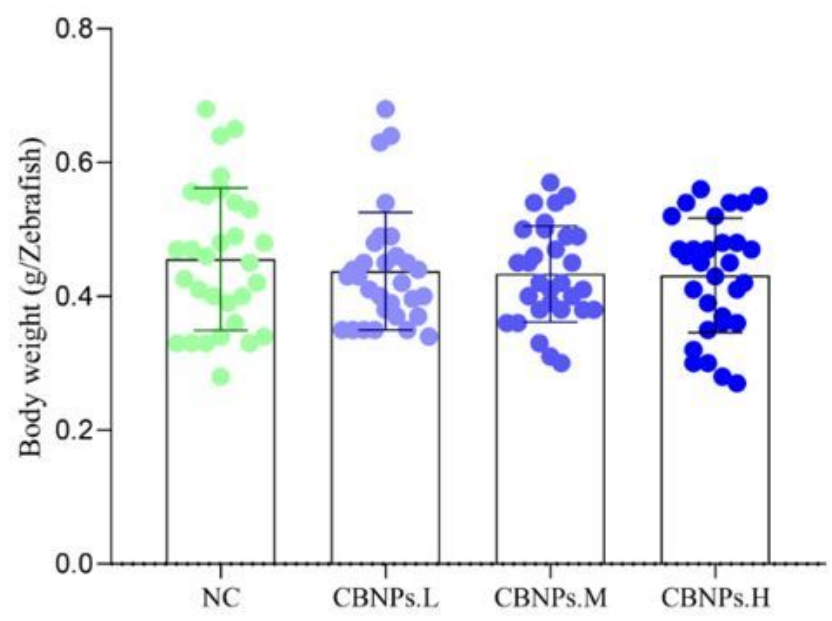

D

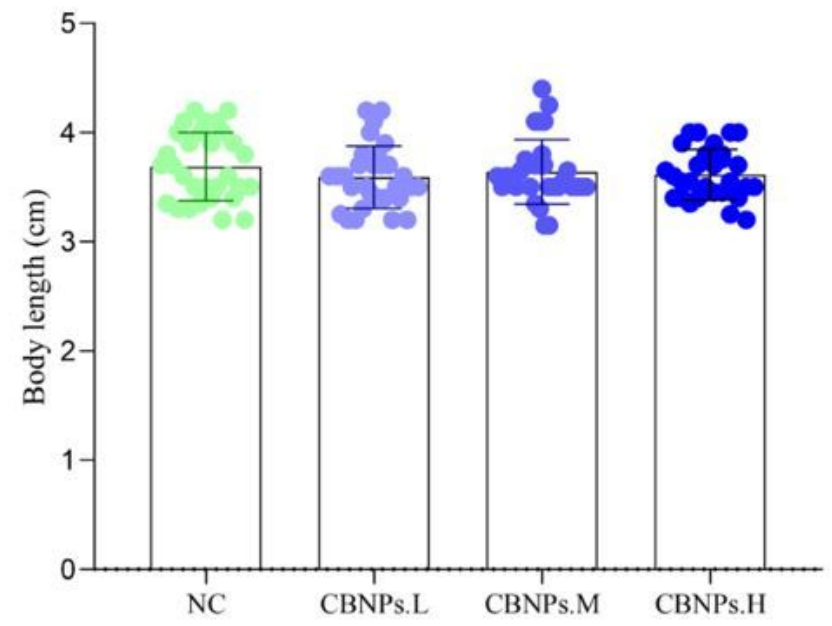

$\mathbf{E}$

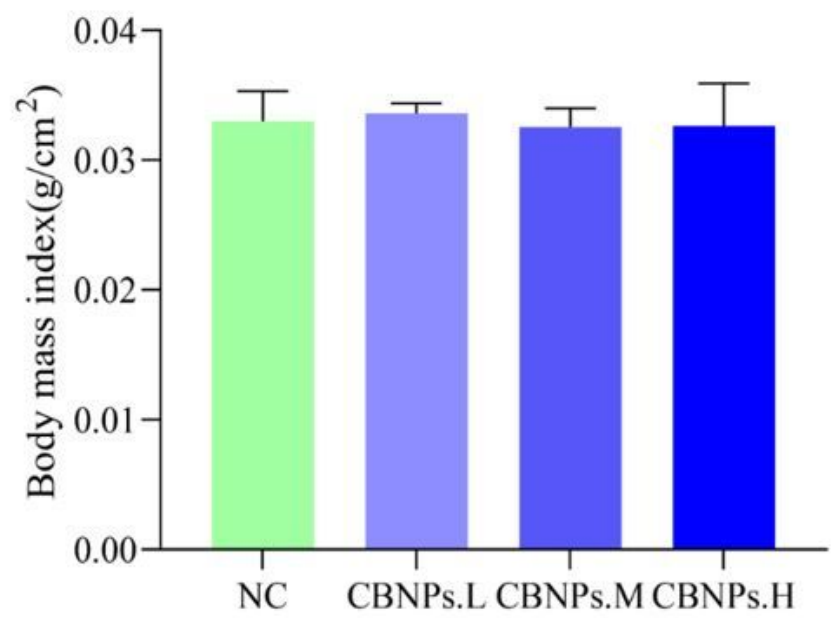

\section{Figure 1}

The effect of CBNPs on the growth status of zebrafish. (A) Typical TEM image of CBNPs (scale bar: $1000 \mathrm{~nm}$; $200 \mathrm{~nm}$ ). (B) Typical SEM image of CBNPs (scale bar: $500 \mathrm{~nm} ; 300 \mathrm{~nm}$ ). (C) Average weight of zebrafish. (D) Average body length of zebrafish. (E) Results of zebrafish body mass index. Zebrafish were exposed to three concentrations of CBNPs water environment for 30 days. All experiments were repeated at least three times, and the results were expressed as mean \pm standard deviation. There were significant differences between two or more groups with different letters, $\mathrm{P}<0.05$; the same letter indicated that there was no significant difference between different groups, $\mathrm{P}>0.05$. 
A
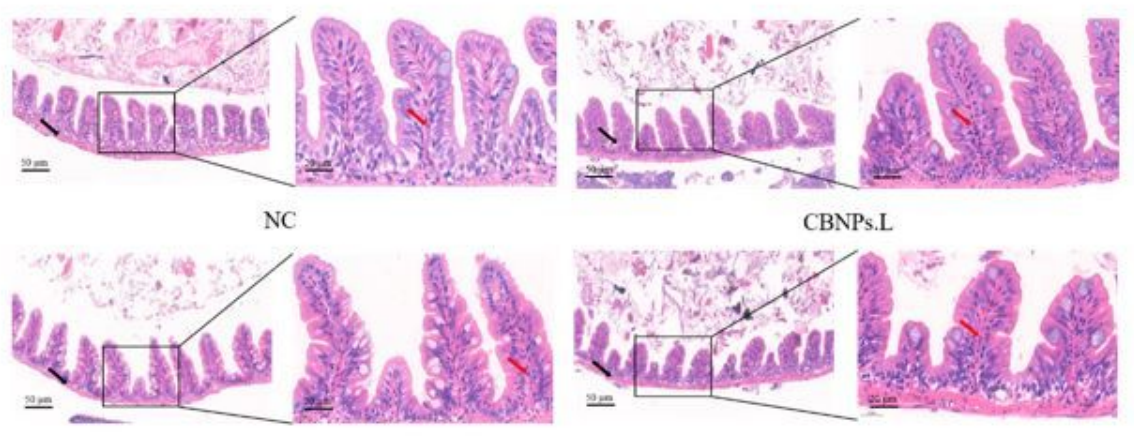

CBNPs.M

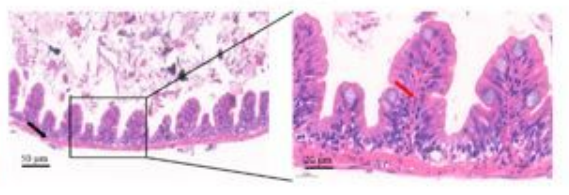

CBNPs.H

B
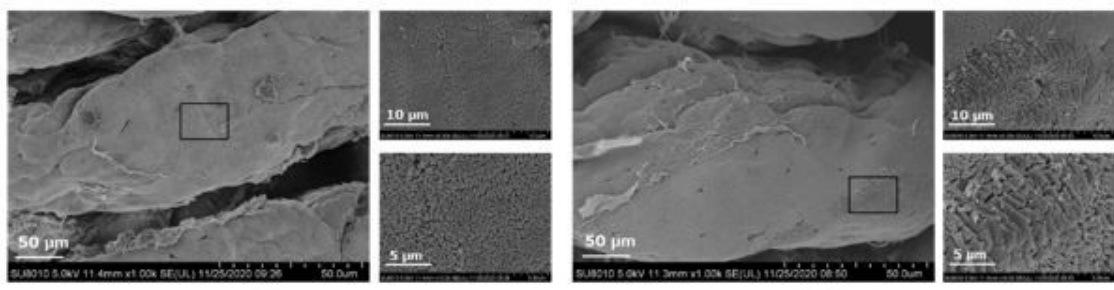

NC

CBNPs.L
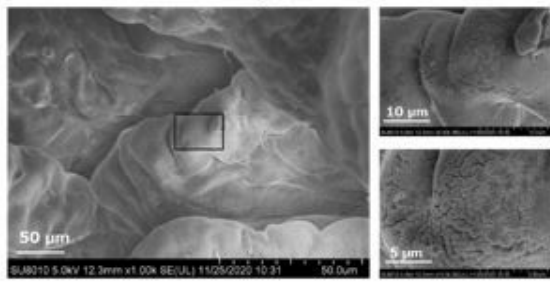

CBNPs.M

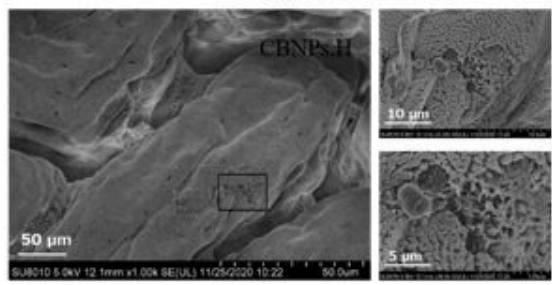

CBNPs.H

C
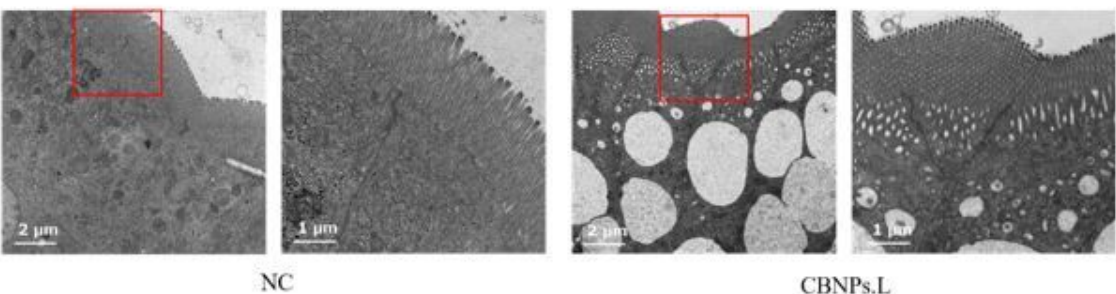

CBNPs.L

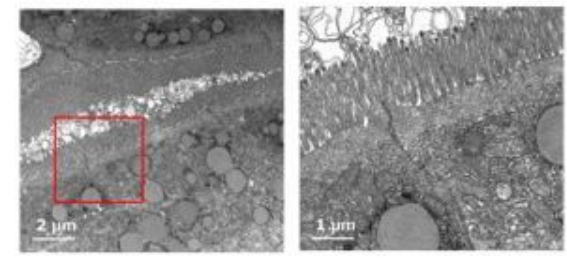

CBNPs.M

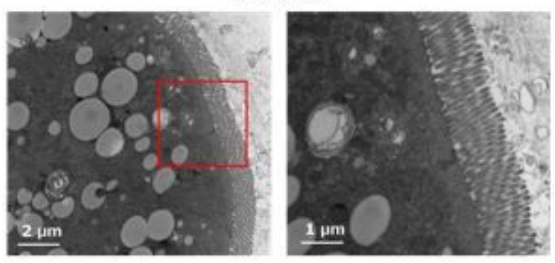

CBNPs.H

\section{Figure 2}

The effect of CBNPs exposure on the structure of zebrafish gut. (A) H\&E staining result of zebrafish intestinal tissue (scale bar: $50 \mu \mathrm{m} ; 20 \mu \mathrm{m}$ ). (B) SEM image of zebrafish intestinal tissue (scale bars: $50 \mu \mathrm{m} ; 10 \mu \mathrm{m} ; 5$ $\mu \mathrm{m})$. (C) TEM image of zebrafish intestinal tissue (scale bar: $2 \mu \mathrm{m}$ ). The zebrafish were exposed to three concentrations of CBNPs in the water environment for 30 days, and all experiments were repeated at least three times. 
A

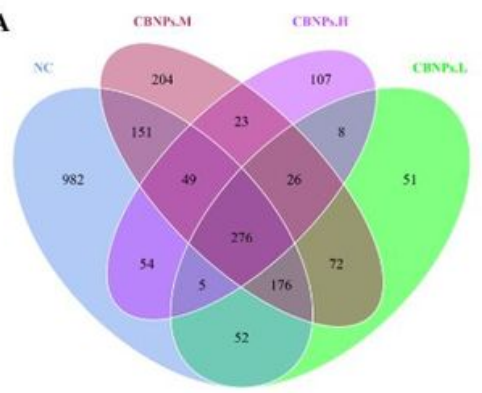

D

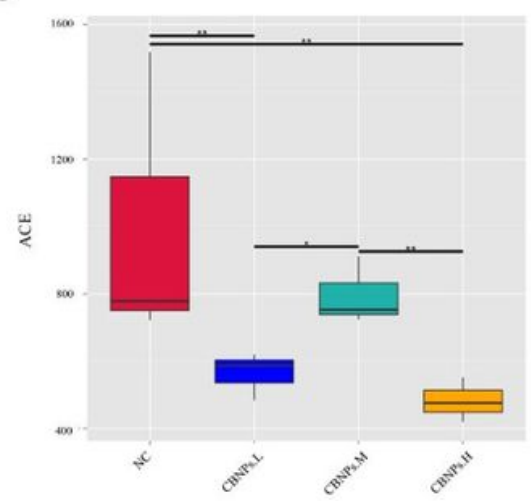

B

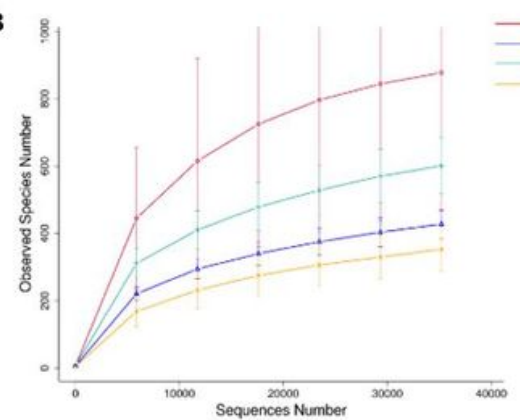

E

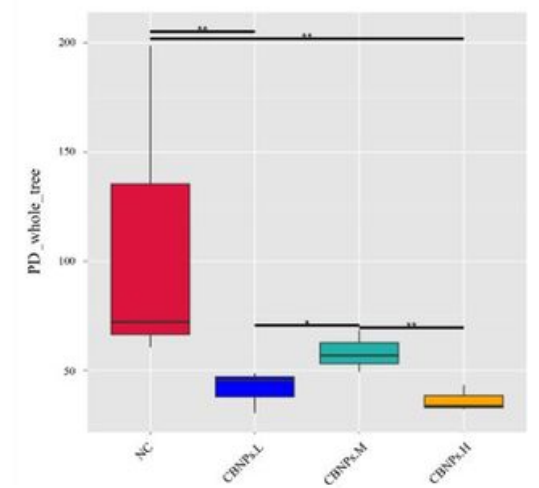

C
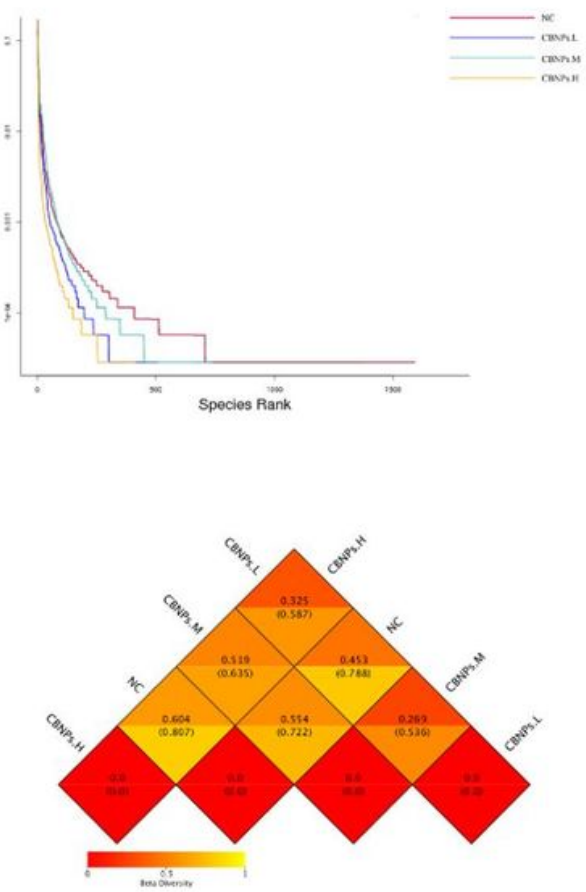

Figure 3

The effect of CBNPs exposure on the diversity of intestinal microflora in zebrafish. The zebrafish were exposed to three concentrations of CBNPs water environment for 30 days, and the intestinal contents were collected for $16 S$ biological information analysis. (A) Venn Graph. (B)Rarefaction Curve and (C)Rank Abundance. (D) Ace index difference box plot between groups and (E) PD_whole_tree index difference box plot between groups. (F) Beta diversity index heat map. All experiments were repeated at least three times, and the results were expressed as mean \pm standard deviation. There were significant differences between two or more groups with different letters, $\mathrm{P}<0.05$; the same letter indicated that there was no significant difference between different groups, $\mathrm{P}>0.05$. 
A

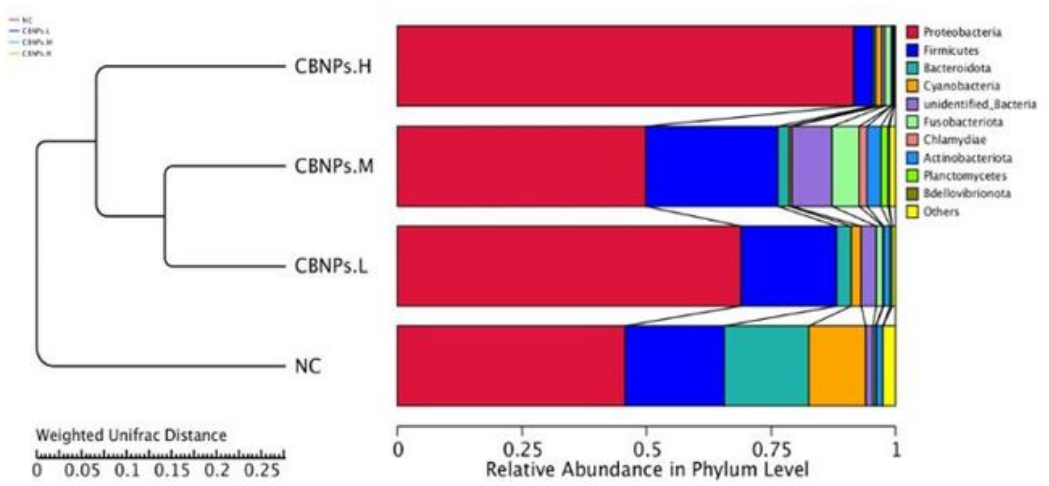

B

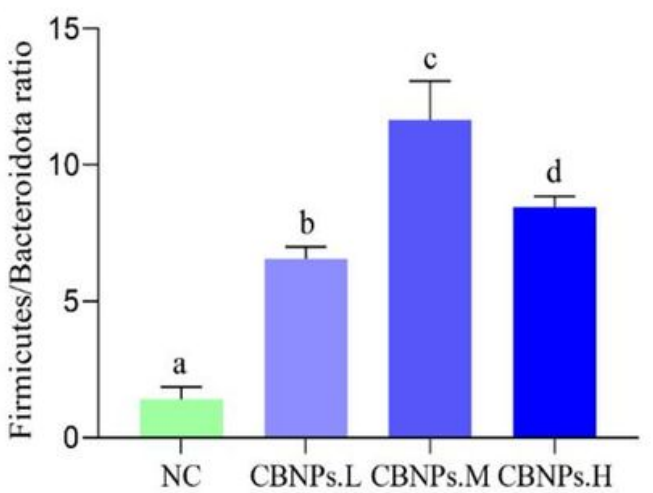

C

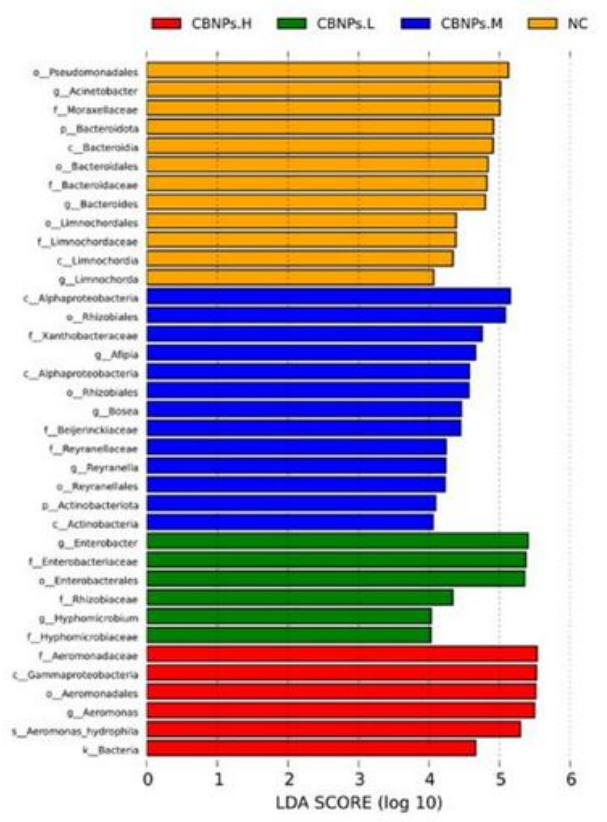

Cladogram
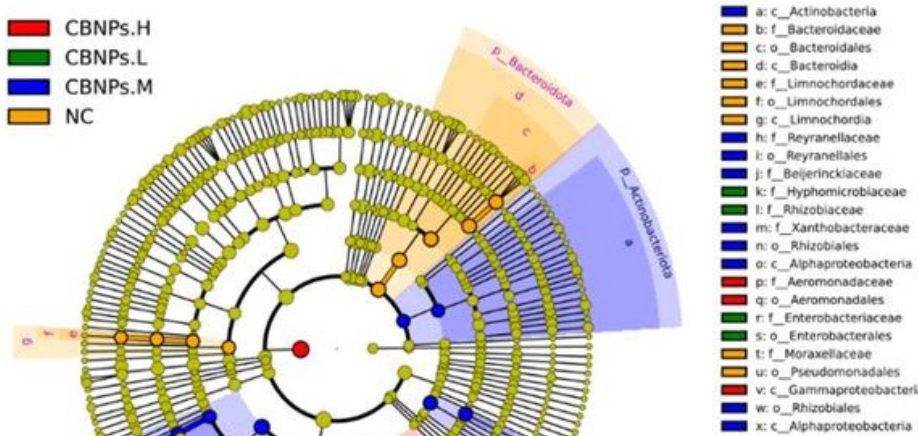

Figure 4

The effect of CBNPs exposure on the composition of zebrafish intestinal flora. The zebrafish were exposed to three concentrations of CBNPs water environment for 30 days, and the intestinal contents were collected for 16S biological information analysis. (A) UPGMA clustering tree based on unweighted Unifrac distance. (B) Bar chart representing the FB ratio. (C) LDA Effect Size results. All experiments were repeated at least three times, and the results were expressed as mean \pm standard deviation. There were significant differences between two or more groups with different letters, $\mathrm{P}<0.05$; the same letter indicated that there was no significant difference between different groups, $\mathrm{P}>0.05$. 
A

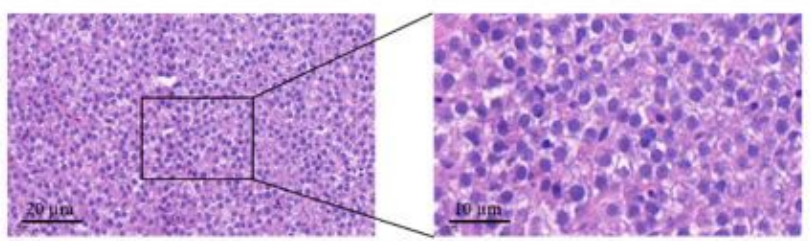

$\mathrm{NC}$

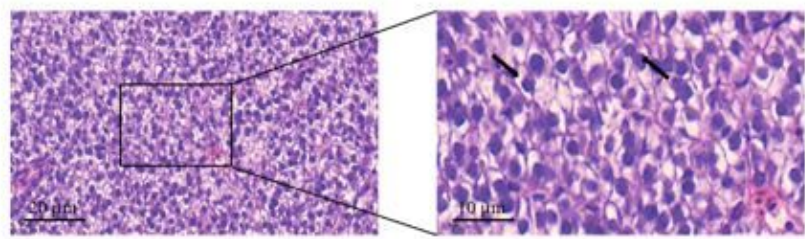

CBNPs.M

B

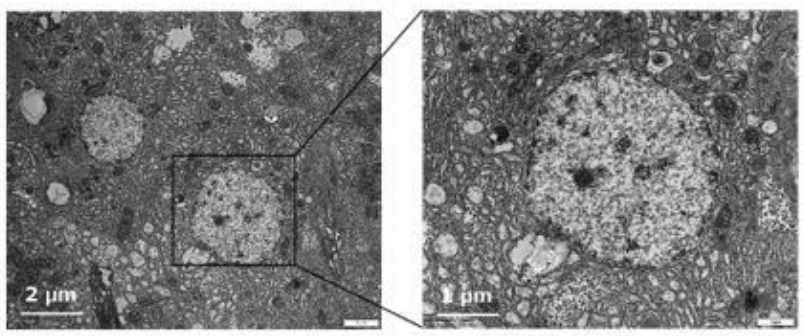

$\mathrm{NC}$

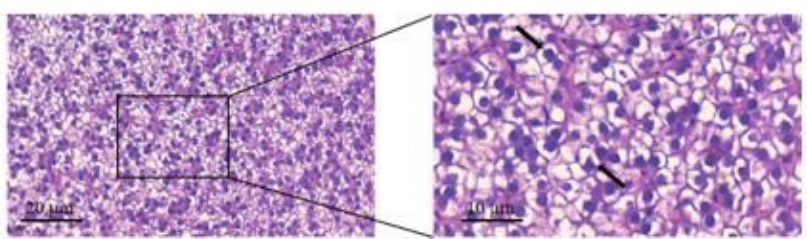

CBNPs.L

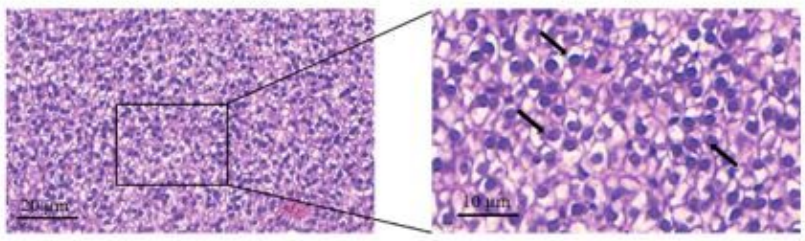

CBNPs.H

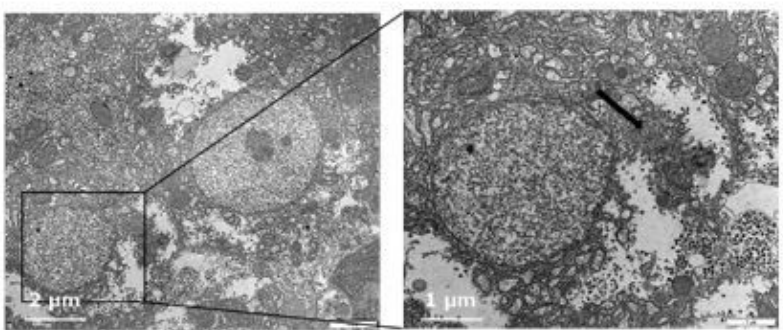

CBNPs.L

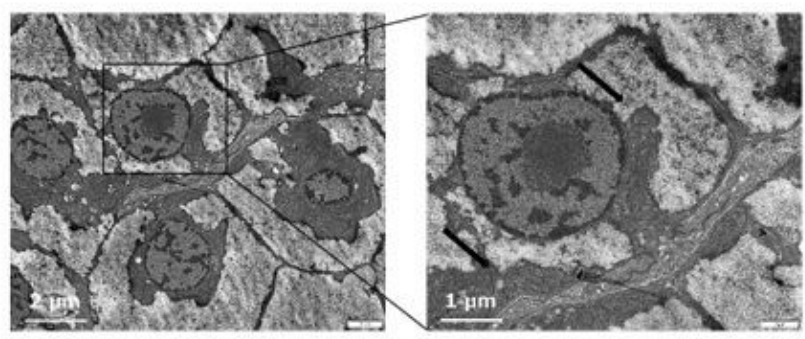

CBNPs.M

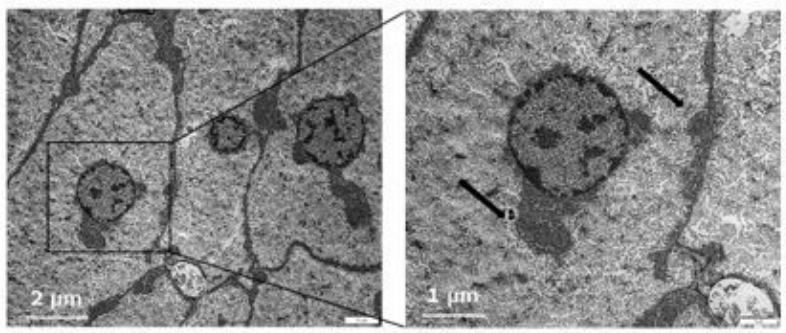

CBNPs.H

C

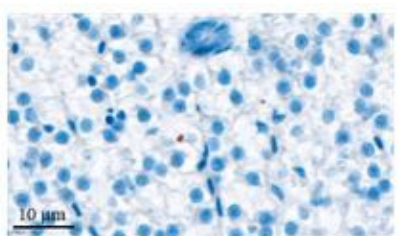

$\mathrm{NC}$

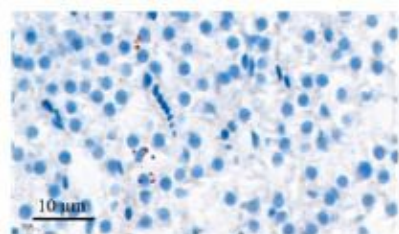

CBNPs.L

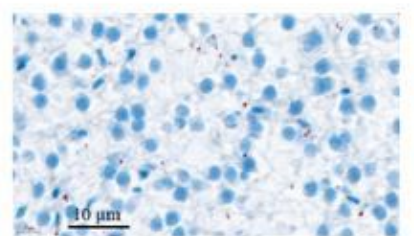

CBNPs.M

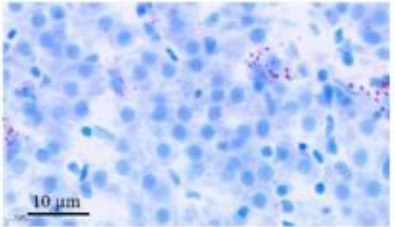

CBNPs.H

\section{Figure 5}

The effect of CBNPs exposure on zebrafish liver. (A) H\&E staining result of zebrafish liver tissue (scale bar: 20 $\mu \mathrm{m} ; 10 \mu \mathrm{m}$ ). (B) TEM image of zebrafish liver tissue (scale bar: $2 \mu \mathrm{m}$ ). (C) Results of oil red 0 staining of zebrafish liver tissue (scale bar: $10 \mu \mathrm{m}$ ). The zebrafish were exposed to three concentrations of CBNPs in the water environment for 30 days, and all experiments were repeated at least three times. 
A

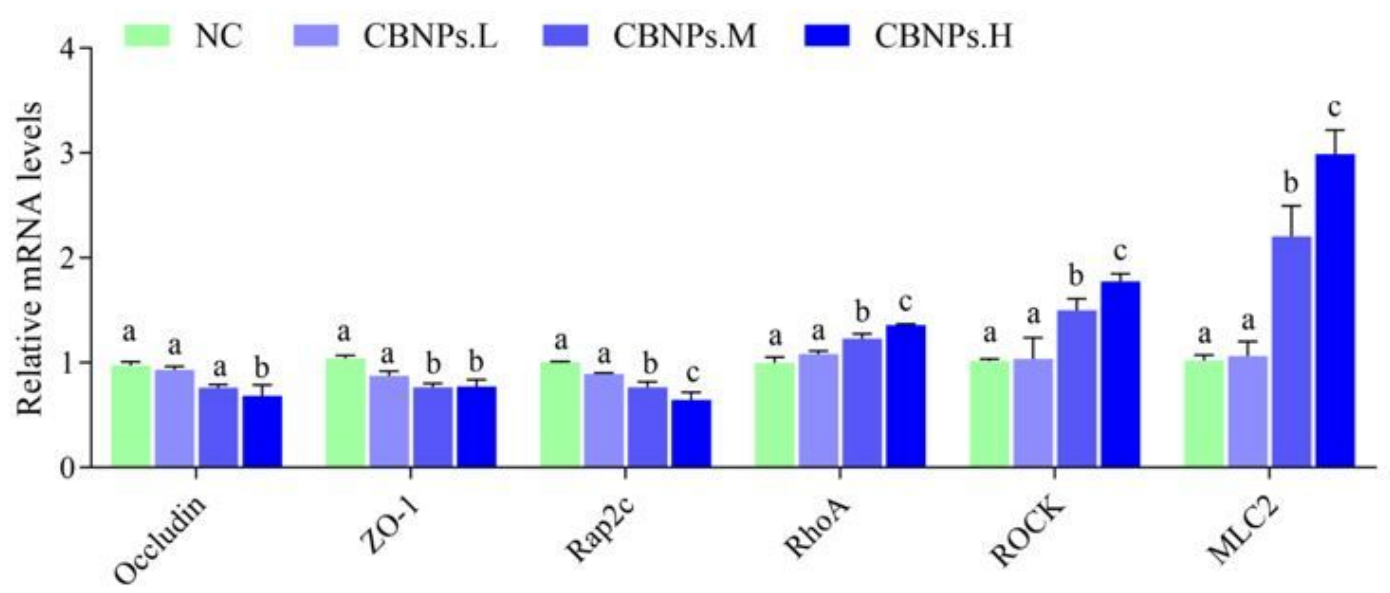

B

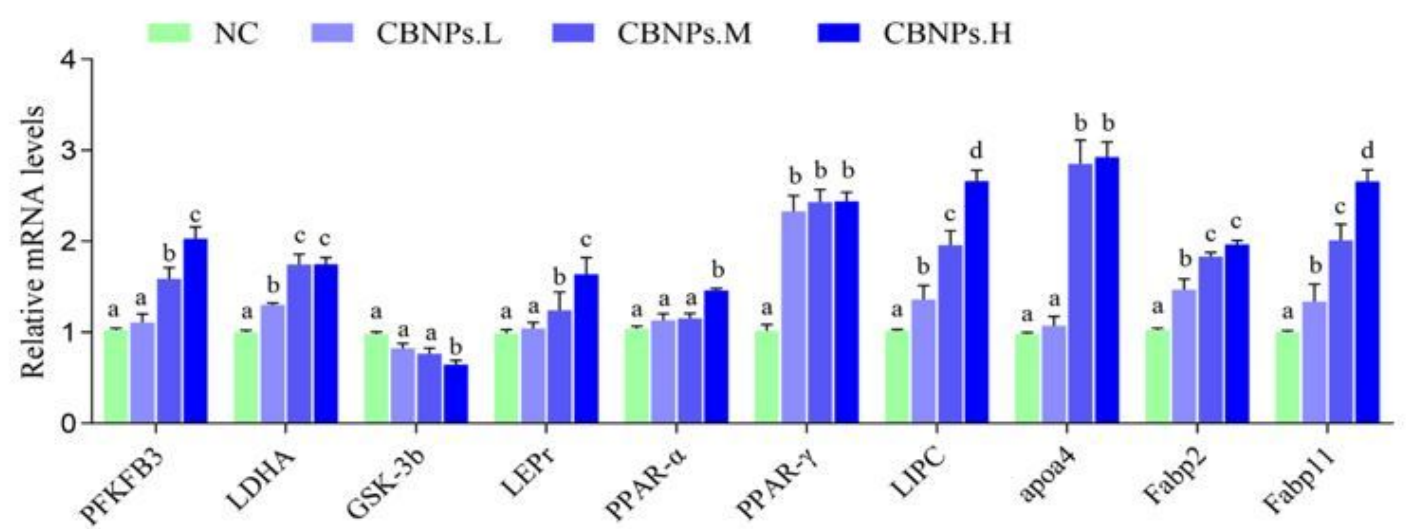

C
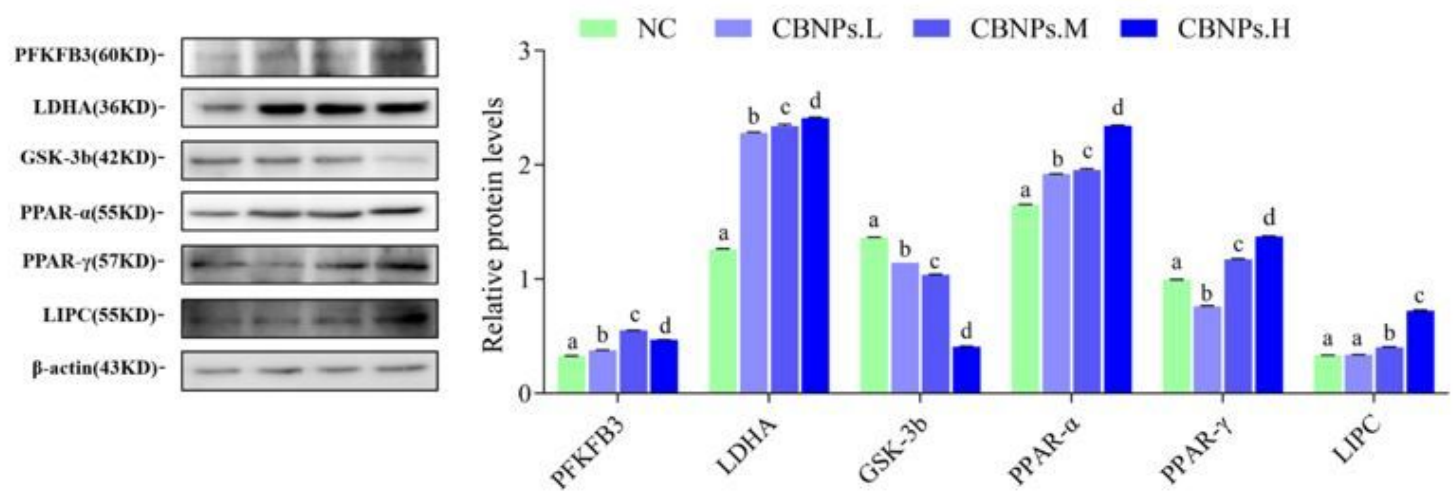

Figure 6

The effect of CBNPs exposure on the expression of genes related to tight junctions and glycolipid metabolism in zebrafish. (A) Results of mRNA expression of tight junction related genes in intestinal tissues. (B) Results of mRNA expression of glycolipid metabolism related genes in liver tissue. (C) Results of protein level expression of genes related to glycolipid metabolism in liver tissues. Zebrafish were exposed to three concentrations of CBNPs water environment for 30 days. All experiments were repeated at least three times, and the results were expressed as mean \pm standard deviation. There were significant differences between two or more groups with 
different letters, $P<0.05$; the same letter indicated that there was no significant difference between different groups, $\mathrm{P}>0.05$.

\section{Supplementary Files}

This is a list of supplementary files associated with this preprint. Click to download.

- GraphicalAbstract.docx 